\title{
How Not to Characterize Planar-emulable Graphs
}

\author{
Markus Chimani $^{1 \star}$, Martin Derka ${ }^{2 \star \star}$, Petr Hliněný ${ }^{2 \star \star \star}$, and Matěj Klusáček ${ }^{2 \star \star \star}$ \\ 1 Algorithm Engineering, Friedrich-Schiller-University Jena, Germany \\ markus.chimani@uni-jena.de \\ 2 Faculty of Informatics, Masaryk University Brno, Czech Republic \\ [hlineny, xderka, xklusac1] @fi.muni.cz
}

\begin{abstract}
We investigate the question of which graphs have planar emulators (a locally-surjective homomorphism from some finite planar graph) - a problem raised already in Fellows' thesis (1985) and conceptually related to the better known planar cover conjecture by Negami (1986). For over two decades, the planar emulator problem lived poorly in a shadow of Negami's conjecture - which is still open - as the two were considered equivalent. But, in the end of 2008, a surprising construction by Rieck and Yamashita falsified the natural "planar emulator conjecture", and thus opened a whole new research field. We present further results and constructions which show how far the planar-emulability concept is from planar-coverability, and that the traditional idea of likening it to projective embeddability is actually very out-of-place. We also present several positive partial characterizations of planar-emulable graphs.
\end{abstract}

\section{Introduction}

A graph $G$ has a planar emulator (cover) $H$ if $H$ is a finite planar graph and there exists a homomorphism from $H$ onto $G$ that is locally surjective (bijective, respectively). In such a case we also say that $G$ is planar-emulable (-coverable). See Def. 2.1 for a precise definition, and Fig. 1 for a simple example. Informally, every vertex of $G$ is represented by one or more vertices in $H$ such that the following holds: Whenever two nodes $v$ and $u$ are adjacent in $G$, any node representing $v$ in $H$ has at least one (in case of an emulator) or exactly one (in case of a cover) adjacent node in $H$ that represents $u$. Conversely, no node representing $v$ in $H$ has a neighbor representing $u$ if $v, u$ are nonadjacent in $G$.

Coarsely speaking, the mutually similar concepts of planar covers and planar emulators both "preserve" the local structure of a graph $G$ while "gaining" planarity for it. Of course, the central question is which nonplanar graphs do have planar covers or emulators.

* M. Chimani has been funded by a Carl-Zeiss-Foundation juniorprofessorship.

* M. Derka has been supported by Masaryk University internal grant for students.

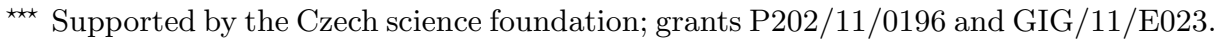



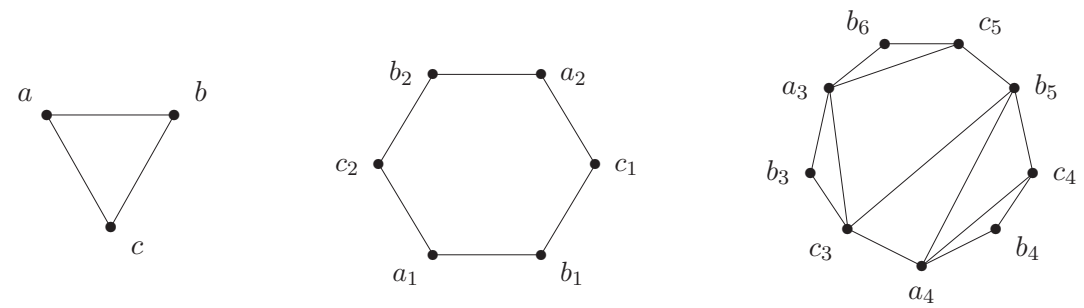

Fig. 1. Examples of a planar cover (center) and a planar emulator (right) of the triangle $G=K_{3}$ (left). We simply denote by $a_{j}, j=1,2, \ldots$ the vertices representing $a$ of $G$, and analogically with $b, c$.

The two concepts emerged independently from works of Fellows $[4,5]$ (emulator) and Negami [15-17] (cover). On the one hand, the class of planar-coverable graphs is relatively well understood. At least, we have the following:

Conjecture 1.1 (Negami [16], 1988). A graph has a (finite) planar cover if and only if it embeds in the projective plane.

Yet, this natural (see below) and firmly believed conjecture is still open today despite of more than 20 years of intensive research. See [10] for a recent survey.

On the other hand, it was no less natural to assume $[4,5]$ that the property of being planar-emulable coincides with planar-coverability. By definition, the latter immediately implies the former. For the other direction, it was highly counterintuitive to assume that, having more than one neighbors in $H$ representing the same adjacent vertex of $G$, could ever help to gain planarity of $H$ - such "additional" edges seem to go against Euler's bound on the number of edges of a planar graph. Hence, it was widely believed:

Conjecture 1.2 (Fellows [5], 1988, falsified 2008). A graph has a (finite) planar emulator if and only if it embeds in the projective plane.

Perhaps due to similarity to covers, no significant effort to specifically study planar-emulable graphs occurred during the next 20 years after Fellows' manuscript [5].

Today, however, we know of one important difference between the two cases: Conjecture 1.2 is false! In 2008, Rieck and Yamashita [18] proved the truly unexpected breakthrough result that there are graphs which have planar emulators, but no planar covers and do not embed in the projective plane; see Theorem 2.4. This finding naturally ignited a new research direction, on which we report herein. We show that the class of planar-emulable graphs is, in fact, much larger than the class of planar-coverable ones; that the concept of projective embeddability seems very out-of-place in the context of planar emulators; and generally, how poorly planar emulators are yet understood.

Apart from its pure graph theoretic appeal, research regarding planar emulators and covers may in fact have algorithmic consequences as well: While 

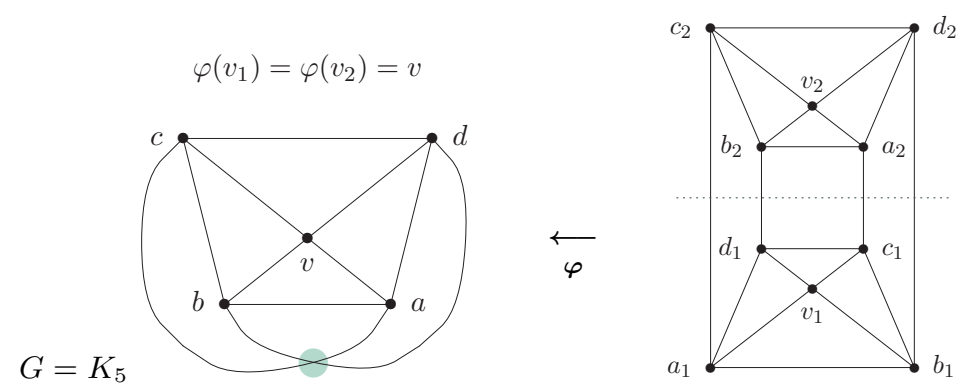

Fig. 2. The graph $G=K_{5}$ (left) and its two-fold planar cover (right) via a homomorphism $\varphi$. The cover is obtained for a "crosscap-less" drawing of $G$ and its mirror image.

Negami's main interest [15] was of pure graph theoretic nature, Fellows [4, and personal communication] considered computing motivation for emulators. Additionally, we would like to sketch another potential algorithmic connection; there are problems that are NP-hard for general graphs, but polynomial-time solvable for planar graphs (e.g., maximum cut), or where the polynomial complexity drops when considering planar graphs (e.g., maximum flow). Yet, the precise breaking point is usually not well understood. Considering such problems for planar-emulable or planar-coverable graphs may give more insight into the problems' intrinsic complexities. Before this can be investigated, however, these classes first have to be reasonably well understood themselves. Our paper aims at improving upon this latter aspect of planar emulators.

This paper is organized as follows: Section 2 discusses all the major prior findings w.r.t. covers and emulators, including the aforementioned result by Rieck and Yamashita. Then, Theorem 2.5 presents our main new improvement. Section 3 reviews some necessary basic properties and tools, most of which have been previously sketched in [5]. In Section 4 we give previously unknown emulator constructions, proving Theorem 2.5 and also showing how unrelated emulators are from covers. We would particularly like to mention a very small and nicely-structured emulator of the notoriously difficult graph $K_{1,2,2,2}$ in Fig. 11. Finally, in Section 5 we study how far one can get in the pursuit to characterize planar-emulable graphs with the structural tools previously used in [11] for covers, and where the current limits are.

\section{On Planar Covers and Emulators}

We restate the problem on a more formal level. All considered graphs are simple, finite, and undirected. A projective plane is the simplest nonorientable surfacea plane with one crosscap (informally, a place in which a bunch of selected edges of an embedded graph may "cross" each other). A graph homomorphism of $H$ into $G$ is a mapping $h: V(H) \rightarrow V(G)$ such that, for every edge $\{u, v\} \in E(H)$, we have $\{h(u), h(v)\} \in E(G)$. 


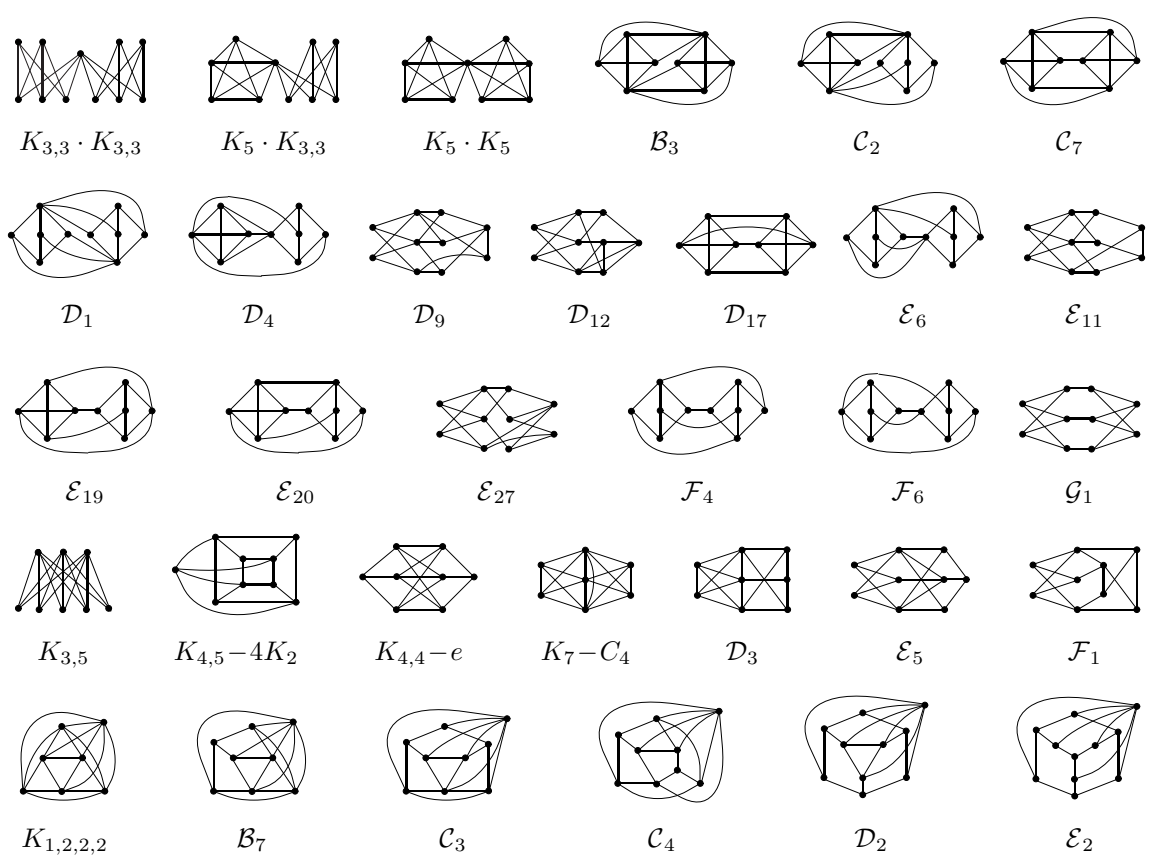

Fig. 3. The 32 connected projective forbidden minors. (The three disconnected ones, $K_{5}+K_{5}, K_{5}+K_{3,3}, K_{3,3}+K_{3,3}$, are skipped since they are not important here.)

Definition 2.1. A graph $G$ has a planar emulator (cover) $H$ if $H$ is a planar finite graph and there exists a graph homomorphism $\varphi: V(H) \rightarrow V(G)$ such that, for every vertex $v \in V(H)$, the neighbors of $v$ in $H$ are mapped by $\varphi$ surjectively (bijectively) onto the neighbors of $\varphi(v)$ in $G$. The homomorphism $\varphi$ is called an emulator (cover) projection.

One immediately obtains the following two claims:

Lemma 2.2. a) If $H$ is a planar cover of $G$, then $H$ is also a planar emulator of $G$. The converse is not true in general.

b) If $G$ embeds in the projective plane, then $G$ has a two-fold planar cover (i.e., $\left|\varphi^{-1}(u)\right|=2$ for all $\left.u \in V(G)\right)$; cf. [15]. See also Fig. 2.

These two claims, together with some knowledge about universal coverings in topology, make Conjectures 1.1 and 1.2 sound very plausible. To precisely describe the motivation for our research direction in planar emulators, we briefly comment on the methods that have been used in the investigation of planarcoverable graphs, too.

Firstly, we note that the properties of planar-coverability and planar-emulability are closed under taking minors (Proposition 3.1), and all 35 minor-minimal nonprojective graphs (projective forbidden minors, Fig. 3) are known [1]. If a connected graph $G$ is projective, then $G$ is planar-coverable (and hence also 

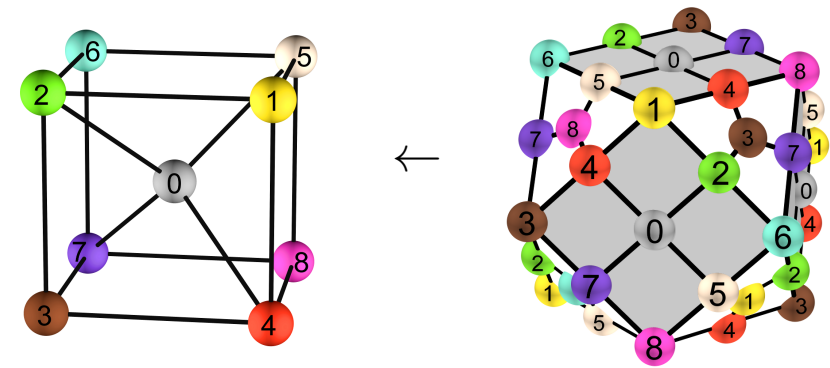

Fig. 4. A colour-coded 3D-rendering of a planar emulator patched on a polyhedral body (right) for the graph $K_{4,5}-4 K_{2}$ (left), taken from http://vivaldi.ics.nara-wu.ac.jp / yamasita/emulator/.

planar-emulable); otherwise, $G$ contains one of the mentioned projective forbidden minors. Hence to prove Conjecture 1.1, only a seemingly simple task remains: we have to show that the known 32 connected projective forbidden minors have no planar covers. The following was established through a series of previous papers:

Theorem 2.3 (Archdeacon, Fellows, Hliněný, and Negami, 1988-98). If the (complete four-partite) graph $K_{1,2,2,2}$ has no planar cover, then Conjecture 1.1 is true.

One can naturally think about applying the same arguments to planar emulators, i.e. to Conjecture 1.2. The first partial results of Fellows [5]- see an overview in Section 3-were, in fact, encouraging. Yet, all the more sophisticated tools (of structural and discharging flavor) used to show the non-existence of planar covers in Theorem 2.3 fail on a rather technical level when applied to emulators. As these problems seemed to be more of technical than conceptual nature, Fellows' conjecture was always believed to be true until the following:

Theorem 2.4 (Rieck and Yamashita [18], 2008). The graphs $K_{1,2,2,2}$ and $K_{4,5}-4 K_{2}$ do have planar emulators (cf. Fig. 4). Consequently, the class of planar-emulable graphs is strictly larger than the class of planar-coverable graphs, and Conjecture 1.2 is false.

We remark that this is not merely an existence result, but the actual (and, surprisingly, not so large) emulators were published together with it. Both $K_{1,2,2,2}$ and $K_{4,5}-4 K_{2}$ are among the projective forbidden minors, and $K_{4,5}-4 K_{2}$ has already been proved not to have a planar cover.

One important new message of our paper is that Theorem 2.4 is not a rarityquite the opposite, many other nonprojective graphs have planar emulators. In particular we prove that, among the projective forbidden minors that have been in doubt since Fellows' [5], all except possibly $K_{4,4}-e$ do have planar emulators:

Theorem 2.5. All of the graphs (Fig. 3) $K_{4,5}-4 K_{2}, K_{1,2,2,2}, \mathcal{B}_{7}, \mathcal{C}_{3}, \mathcal{C}_{4}, \mathcal{D}_{2}$, $\mathcal{E}_{2}$, and also $K_{7}-C_{4}, \mathcal{D}_{3}, \mathcal{E}_{5}, \mathcal{F}_{1}$ have planar emulators. 
Consequently, the class of planar-emulable graphs is much larger than the class of planar-coverable ones. We refer to Section 4 for details.

\section{Basic Properties of Emulators}

In this section, we review the basic established properties of planar-emulable graphs. These are actually all the properties of planar-coverable graphs which are known to extend to planar emulators (though, the extensions of some of the proofs are not so straightforward).

The claims presented here, except for Theorem 3.6, were proved or sketched already in the manuscript [5] of Fellows. However, since [5] has never been published, we consider it appropriate to include their full proofs.

We begin with two crucial closure properties.

Proposition 3.1 (Fellows [5]). The property of being planar-emulable is closed under taking minors; i.e., under taking subgraphs and edge contractions.

Proof. Let $G$ be a planar-emulable graph, and planar $H$ be its emulator via a projection $\varphi$. We prove this easy proposition by showing how $H$ is modified to accommodate for the elementary reduction steps in $G$; vertex/edge deletion, and edge contraction. Say, if a vertex $v \in V(G)$ is deleted, then also all vertices $\varphi^{-1}(v)$ representing $v$ are deleted from $H$.

An edge $f=x y \in E(H)$ represents the edge $e \in E(G)$ if $e=\{\varphi(x), \varphi(y)\}$. Whenever an edge $e \in E(G)$ is deleted, so are all the edges representing $e$ in $H$. Lastly, if an edge $e \in E(G)$ is contracted, then every component induced by the edges representing $e$ in $H$ is also contracted into a single vertex (note that such components may contain more than one edge representing $e$ in the case of an emulator), and possible parallel edges are simplified. All these operations preserve planarity of $H$, and the outcome is an emulator of the graph resulting from $G$.

Proposition 3.2 (Fellows [5]). The property of being planar-emulable is closed under applying $Y \Delta$-transformations; i.e., the operations replacing (successively) any degree-3 vertex with a triangle on its three neighbors.

Proof. Let $G$ be a planar-emulable graph and $v \in V(G)$ a vertex of degree 3 . Denote by $G^{\prime}$ the graph obtained from $G$ by applying the $Y \Delta$-transformation of $v$. Suppose a planar graph $H$ that is an emulator of $G$ via a projection $\varphi$.

In the (optimistic) case that all the vertices of $H$ in $\varphi^{-1}(v)$ are also of degree 3 , we simply successively apply $Y \Delta$-transformations to all the vertices in $\varphi^{-1}(v)$ (which form an independent set of $H$ ), and the resulting graph $H^{\prime}$ will be again planar and an emulator of $G^{\prime}$.

It remains to justify our optimistic assumption about degree- 3 vertices in $\varphi^{-1}(v)$ of a suitable planar emulator $H$ of $G$, which follows from the following claim applied to $X=\{v\}$ : 

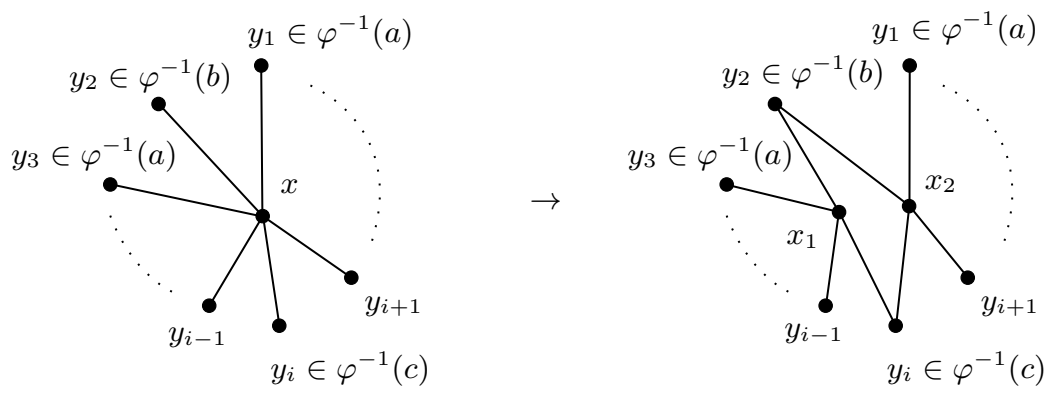

Fig. 5. Splitting vertex $x$ with a cubic image into vertices of lower degree.
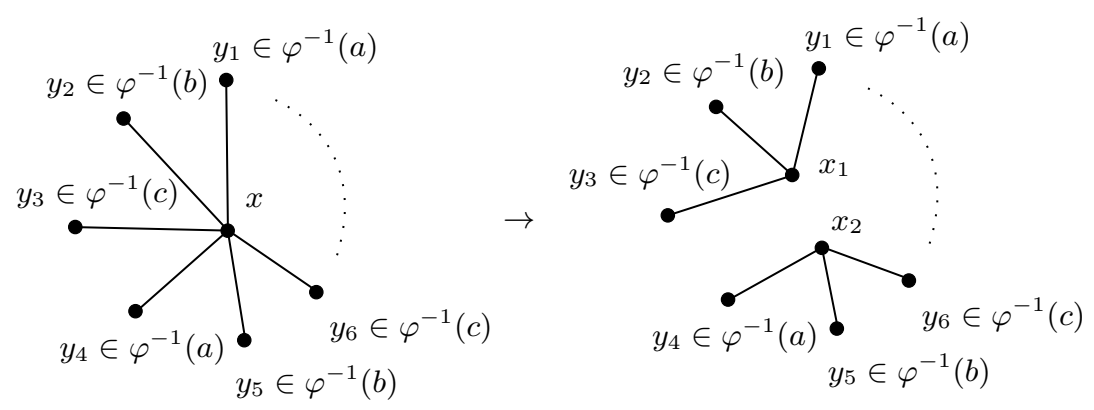

Fig. 6. Illustration of the last case of the proof of Lemma 3.3.

Lemma 3.3 (Fellows [5]). Let $G$ be a planar-emulable graph and $X \subseteq V(G)$ an independent set of vertices of degree 3. Then there exists a planar emulator $H$ of $G$ with a projection $\varphi: V(H) \rightarrow V(G)$ such that every vertex $u \in \varphi^{-1}(v)$ over all $v \in X$ is of degree 3 .

Proof. Whenever $F$ is an emulator of our graph $G$ with a projection $\psi: V(F) \rightarrow$ $V(G)$; let $D g(F)(\geq 3)$ shortly denote the maximal $F$-degree of the vertices $u \in \psi^{-1}(v)$ over all $v \in X$. We choose $H$ as a planar emulator of $G$ with projection $\varphi$ such that the value $D g(H)$ is minimized.

Assume, for a contradiction, that $D g(H)>3$, and choose any vertex $x \in$ $\varphi^{-1}(v)$ where $v \in X$ such that $x$ is of $H$-degree $D g(H)=d>3$. Let $a, b, c$ be the three neighbors of $v$ in $G$. We denote by $w$ the circular word of length $d$ over the alphabet $\{a, b, c\}$ formed of the letters $\varphi\left(y_{1}\right) \varphi\left(y_{2}\right) \ldots \varphi\left(y_{d}\right)$, where $y_{1}, \ldots, y_{d}$ are the neighbors of $x$ in $H$ in this cyclic order. Then, one of the following three cases, up to symmetry, occurs in $w$ :

- $w$ contains a subword aa: By merging the corresponding two vertices of $H$ representing $a$ into one, the degree of $x$ drops to $d-1$.

- $w$ contains a subword $a b a$ : Without loss of generality, it is $\varphi\left(y_{1}\right)=\varphi\left(y_{3}\right)=a$, $\varphi\left(y_{2}\right)=b$, and $\varphi\left(y_{i}\right)=c$ for some $4 \leq i \leq d$ (to be a valid emulator of $G$ ). 


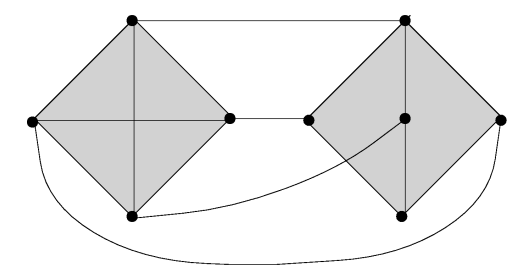

Fig. 7. An example of a graph having two disjoint k-graphs (shaded in gray).

We modify $H$ by splitting vertex $x$ into $x_{1}, x_{2}$ with $\varphi\left(x_{1}\right)=\varphi\left(x_{2}\right)=\varphi(x)$, so that $x_{1}$ is adjacent to $y_{2}, y_{3}, \ldots, y_{i}$ and $x_{2}$ to $y_{i}, \ldots, y_{d}, y_{1}, y_{2}$; see Fig. 5 . Clearly, the degrees of $x_{1}, x_{2}$ are now smaller than $d$.

- $w=(a b c)^{+}$: Then $H$ may be modified as shown in Fig 6 , and the degrees of the newly created vertices drop down to 3 .

In each of the cases it is easy to see that the obtained graph $H^{\prime}$ is still a valid planar emulator of $G$, and that only degrees of some neighbors of $x$ in $H$ could have gone up from $H$ to $H^{\prime}$. Hence, as $X$ is an independent set, we can repeat the above construction for all the vertices $x \in \varphi^{-1}(X)$ (which form an independent set in $H$, too) of degree $d$, and in finitely many steps obtain a contradiction to minimality of $D g(H)$.

Next, we identify some easy forbidden minors for planar-emulable graphs among the known list of projective forbidden minors (cf. Lemma $2.2 \mathrm{~b}$ ). Again, these extend folklore knowledge about planar-coverable graphs.

We say that a graph $G$ contains two disjoint $k$-graphs if there exist two vertexdisjoint subgraphs $J_{1}, J_{2} \subseteq G$ such that, for $i=1,2$, the graph $J_{i}$ is isomorphic to a subdivision of $K_{4}$ or $K_{2,3}$, the subgraph $G-V\left(J_{i}\right)$ is connected and adjacent to $J_{i}$, and contracting in $G$ all the vertices of $V(G) \backslash V\left(J_{i}\right)$ into one results in a nonplanar graph (i.e. containing a $K_{5^{-}}$or $K_{3,3}$-subdivision). We remark that such $G$ is always nonprojective [6]. See an example in Fig. 7 .

Theorem 3.4 (Fellows [5]). A planar-emulable graph $G$ cannot contain two disjoint k-graphs. Consequently, each of the 19 graphs-projective forbidden minors - in the first three rows of Fig. 3 has no planar emulator.

Proof. Suppose, for a contradiction, that $G$ contains two disjoint k-graphs $J_{1}, J_{2} \subseteq G$, and that there exists a planar emulator $H$ with a projection $\varphi: V(H) \rightarrow V(G)$. Let $H_{i}, i=1,2$, denote the subgraph of $H$ induced by the edges representing $E\left(J_{i}\right)$ in the projection $\varphi$. (An edge $f=x y \in E(H)$ represents $e$ if $e=\{\varphi(x), \varphi(y)\}$.) Then $H_{1}$ and $H_{2}$ are vertex-disjoint, and up to symmetry between $H_{1}, H_{2}$, there exists a component $A_{1} \subseteq H_{1}$ such that all other components of $H_{1}, H_{2}$ lie in the outer face of $A_{1}$ in the plane drawing of $H$.

Since $G-V\left(J_{1}\right)$ is connected and adjacent to $J_{1}$, it follows that all the vertices of $V(H) \backslash V\left(A_{1}\right)$ lie in the outer face of $A_{1}$. So, by contracting $V(H) \backslash V\left(A_{1}\right)$ into one vertex $x$ we obtain a planar graph $H_{0}$ which is an emulator of the nonplanar 
graph $G_{0}$ resulting from $G$ by contracting all $V(G) \backslash V\left(J_{1}\right)$ into one vertex $w$. Let $\varphi_{0}: V\left(H_{0}\right) \rightarrow V\left(G_{0}\right)$ be the derived emulator projection. Then $\varphi_{0}^{-1}(w)=\{x\}$, which is a contradiction to further Lemma 3.5.

Lemma 3.5 (Fellows [5]). In every planar emulator $H$ of a nonplanar connected graph $G$ with the projection $\varphi: V(H) \rightarrow V(G)$, the following holds: $\left|\varphi^{-1}(v)\right| \geq 2$ for each $v \in V(G)$.

Proof. Suppose, for a contradiction, that $\varphi^{-1}(w)=\{x\}$ for some $w \in V(G)$ and $x \in V(H)$. Firstly, we prove the claim for $G=K_{5}$ : Then $H-x$ is an emulator of $K_{4}=K_{5}-w$, and $H-x$ is outerplanar, i.e. all its vertices are incident with one face since they are all adjacent to the same vertex $x$ in $H$. However, all degrees in $H-x$ are at least 3 while an outerplanar simple graph must contain a vertex of degree $\leq 2$, a contradiction.

Secondly, we consider $G=K_{3,3}$ which is a bit more complicated case. Then $H-x$ is an emulator of $G-w=K_{2,3}$. Obviously, $H-x$ may be assumed connected. Let $B$ be a leaf block of $H-x$, i.e. a maximal 2-connected subgraph of $H-x$ such that $B$ shares only (at most) one vertex with the rest of $H-x$. Let $\{a, b, c\} \subseteq V\left(K_{2,3}\right)$ denote the unique independent set of size three, and $\{s, t\} \subseteq$ $V\left(K_{2,3}\right)$ be the other two vertices. Then every vertex of $H-x$ representing $s$ or $t$ is of degree $\geq 3$, and there exists such $y \in V(B)$ having all neighbors $z_{1}, z_{2}, z_{3}$ in $B$. Since $z_{1}, z_{2}, z_{3}$ are mapped to $a, b, c$, they must all be adjacent to $x$ in plane $H$, which contradicts 2-connectivity of $B$.

Third, we consider any other nonplanar graph $G$, i.e. containing a minor isomorphic to $K_{5}$ or $K_{3,3}$. Notice in the proof of Proposition 3.1 that even a minor $G^{\prime}$ of $G$ will have an emulator $H^{\prime}$ (a minor of $H$ ) with projection $\varphi^{\prime}$ such that $\left|\varphi^{\prime-1}\left(w^{\prime}\right)\right|=1$ for $w^{\prime}$ corresponding to original $w$. Hence we are finished by one of the previous two cases.

Finally, we include the following sporadic result which seems to be just a very fortunate extension of the cover case, heavily benefiting from Lemma 3.3.

Theorem 3.6 (Fellows / Huneke [12]). The graph $K_{3,5}$ has no planar emulator.

Proof. Suppose, for a contradiction, that the graph $K_{3,5}$ has a planar emulator $H$ with a projection $\varphi: V(H) \rightarrow V\left(K_{3,5}\right)$, and denote by $X \subseteq V\left(K_{3,5}\right)$ the subset of degree-3 vertices in $K_{3,5}$. By Lemma 3.3 , we may assume that all the vertices in $H$ representing some vertex of $X$ are of degree 3 as well. Furthermore, since a homomorphic image of an odd cycle contains an odd cycle but $K_{3,5}$ is bipartite, the emulator $H$ is also bipartite. Hence the overall setting is (almost) as in the cover case and we may apply arguments analogical to $[12,10]$.

We use the so called discharging method. We assign charge of $3(4-\operatorname{deg}(x))$ to every vertex $x$ of degree $\operatorname{deg}(x)$, and of $3(4-\operatorname{len}(f))$ to every face $f$ of length $\operatorname{len}(f)$ in $H$. Note that $\operatorname{len}(f) \geq 4$ is always even in $H$. By Euler's formula, the total charge of $H$ is positive $12 \cdot 2>0$. The aim of the discharging method is to redistribute this charge across $H$ in a way that the resulting amount is nonpositive, which would give a contradiction to supposed planarity of $H$. 


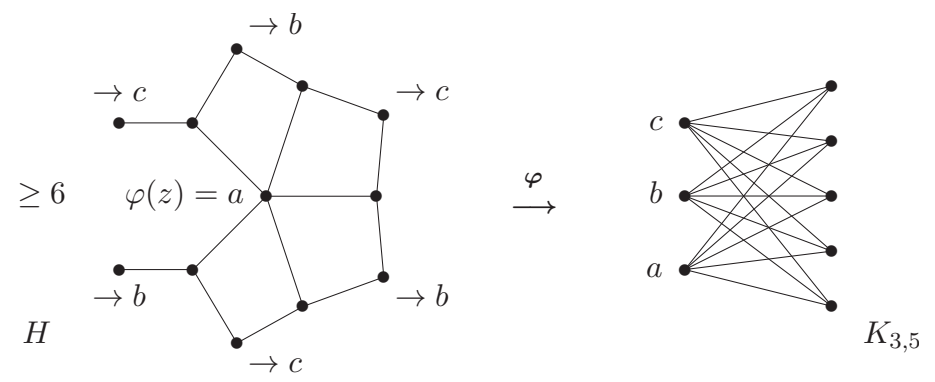

Fig. 8. An illustration of the proof of Theorem 3.6; not all faces incident with the central vertex $z$ can be of length 4 .

Subsequently, every degree-3 vertex of $H$ (i.e., every vertex representing one of $X$ ) sends its charge equally 1 to each neighbor. Then any vertex $y \in V(H)$ of degree $d \geq 6$ or more ends up with total charge of at most $3(4-d)+d=$ $12-2 d \leq 0$. On the other hand, every degree- 5 vertex $z \in V(H)$ now has charge of $-3+5=2$. That charge is subsequently sent from $z$ to any incident face of length $\ell \geq 6$ in $H$, which then ends up with charge of at most $3(4-\ell)+\ell=12-2 \ell \leq 0$. This gives the required contradiction provided we can show that not all faces incident with $z$ are of length 4 .

Now assume the latter, and denote by $\{a, b, c\}=V\left(K_{3,5}\right) \backslash X$ such that $\varphi(z)=a$. See Fig. 8. The neighbors of $z$ in $H$ are all of degree 3, and each one of them needs one additional neighbor representing $b$ and one representing $c$. So the vertices in the second neighborhood of $z$ in $H$ alternatingly represent $b, c, b, c, \ldots$, and we thus cannot have exactly five of them incident to faces of length 4 around $z$. This contradiction proves that some face incident to $z$ is of length $\geq 6$, as needed to finish the proof.

Lastly, we remark that also the graphs $K_{7}$ and $K_{4,4}$ cannot have planar emulators by Euler's formula, but these may not be minor-minimal such ones. In particular, there is some (yet unknown) subgraph of the complete graph $K_{7}$ which is a minor-minimal non-planar-emulable graph, as discussed in Section 6 .

\section{Constructing New Planar Emulators}

The central part of this paper deals with new constructions of planar emulators which consequently give the proof of Theorem 2.5. We remark that, to our best knowledge, no planar emulators of nonprojective graphs other than those mentioned in Theorem 2.4 have been studied or published prior to our paper. Moreover, using our systematic techniques we have succeeded in finding a much smaller emulator for $K_{1,2,2,2}$ than the one presented by Rieck and Yamashita in [18].

Planar emulator for $\mathcal{E}_{2}$. In order to obtain an easily understandable description of an emulator for $\mathcal{E}_{2}$, we note the following: A graph isomorphic to $\mathcal{E}_{2}$ (in 

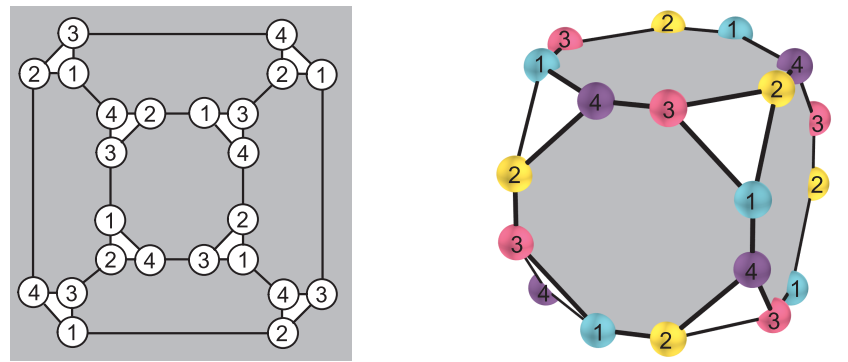

Fig. 9. A planar emulator (actually, a cover) for the complete graph $K_{4}$ with the rich faces depicted in gray colour. The same figure in a "polyhedral" manner on the right.

Fig. 3) can be constructed from the complete graph $K_{4}$ on $V\left(K_{4}\right)=\{1,2,3,4\}$ by subdividing each edge once, calling the new vertices bi-vertices, and finally introducing a new vertex 0 adjacent to all the bi-vertices.

A similar sketch can be applied to a construction of a planar emulator for $\mathcal{E}_{2}$ : If one can find a planar emulator for $K_{4}$ with the additional property that each edge is incident to at least one rich face - i.e., a face bordered by representatives of all edges of $K_{4}$, then a planar emulator for $\mathcal{E}_{2}$ can be easily derived from this. More precisely, if $H_{0}$ is such a special emulator of $K_{4}$, see an example in Fig. 9, then the following construction is applied. Each edge of $H_{0}$ is subdivided with a new vertex representing the corresponding bi-vertex of $\mathcal{E}_{2}$, and a new vertex representing the vertex 0 of $\mathcal{E}_{2}$ is added to every rich face of $H_{0}$ such that it is adjacent to all the subdividing vertices within this face. The resulting plane graph $H$ clearly is an emulator for $\mathcal{E}_{2}$ (and this construction is reversible).

Perhaps the simplest possible such an emulator for $K_{4}$ with rich faces is depicted in Fig. 9 (left). This leads to the nicely structured planar emulator for the graph $\mathcal{E}_{2}$ in Fig. 10. It is also worth to note that the same core ideas which helped us to find this emulator for $\mathcal{E}_{2}$, were actually used in [9] to prove the nonexistence of a planar cover for $\mathcal{E}_{2}$. This indicates how different the coverability and emulability concepts are from each other, too.

There is another interesting point to mention about our emulator for $\mathcal{E}_{2}-$ the plane graph can be quite beautifully pictured as a polyhedron (compare to Fig. 9 right). Consider a cube; it has 8 corners, 12 ridges (we avoid the term edge here), and 6 facets. Interpreting the corners, ridges, and facets of any convex polyhedron as the vertices, edges, and faces gives a planar graph; geometrically, we obtain a plane drawing of the cube graph by choosing a perspective projection from a point close to one of the cube facets.

Then we may truncate ("cut") each of the eight corners of the cube (geometrically, to obtain a truncated hexahedron, an Archimedean solid), and represent each of the eight 6-cycle (but triangle-shaped) faces of the emulator from Fig. 10 at each of the truncated corners. We place, among those 6-faces, pairs of the same type at the opposite corners of the cube. Then we add the respective missing edges along the cube ridges, and finally we place the remaining vertices 


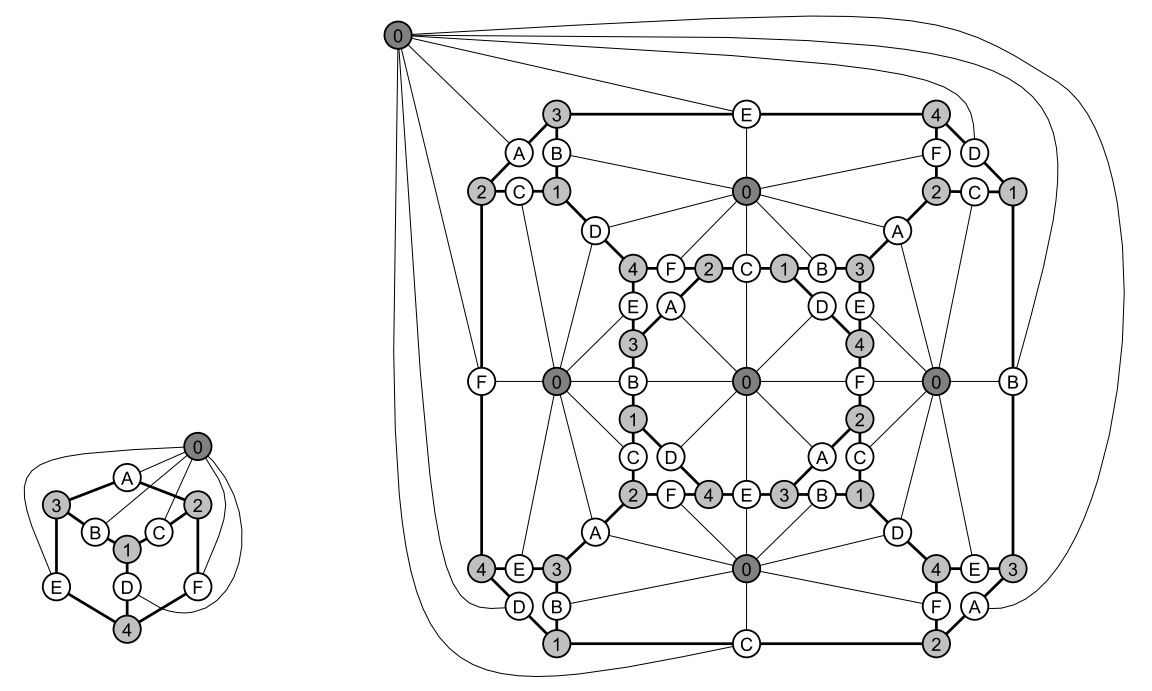

Fig. 10. A planar emulator for $\mathcal{E}_{2}$. The bi-vertices of the construction are in white and labeled with letters, while the numbered core vertices (cf. Fig. 9) are in gray.

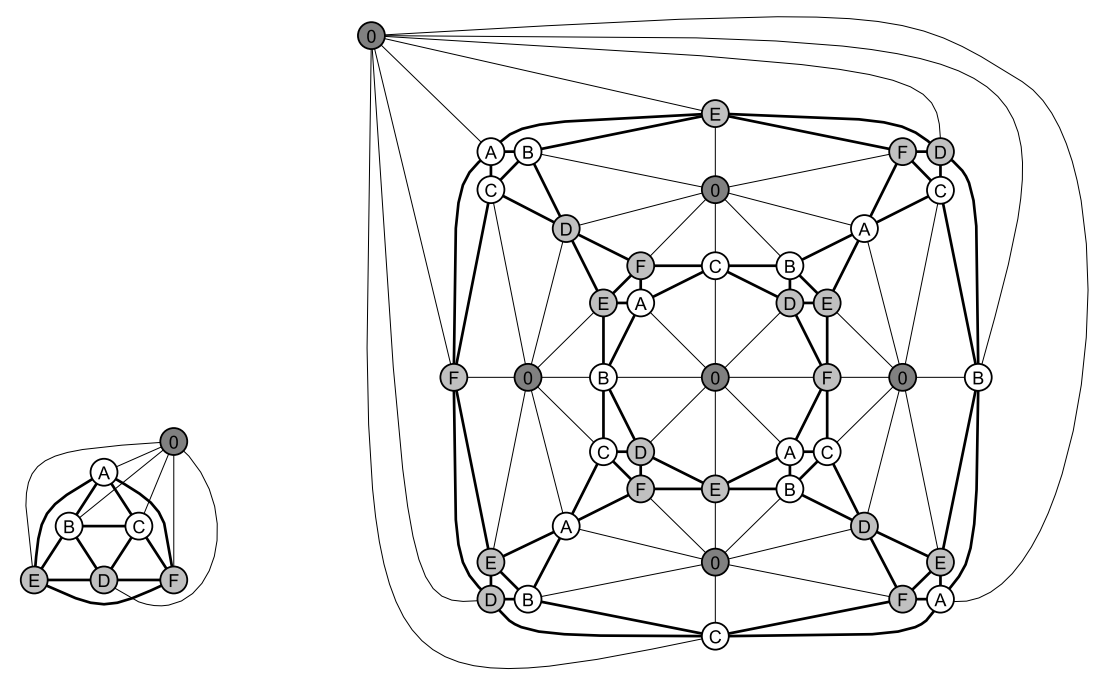

Fig. 11. A planar emulator for $K_{1,2,2,2}$; obtained by taking $Y \Delta$-transformations on the core vertices labeled $1,2,3,4$ of the $\mathcal{E}_{2}$ emulator from Fig. 10.

representing 0 into the six octagonal facets of the body, which correspond to the rich gray faces from Fig. 9. 
More emulators derived from the $\mathcal{E}_{\mathbf{2}}$ case. By Proposition 3.2, the property of having a planar emulator is closed under taking $Y \Delta$-transformations. Moreover, the proof is constructive, and we may use it to mechanically produce new emulators from existing ones (this principle goes even slightly beyond straightforward $Y \Delta$-transformations, see Section 5). Therefore we can easily obtain an alternative emulator for $K_{1,2,2,2}$ (cf. Theorem 2.4) which is significantly smaller and simpler than the original one in [18]. The emulator is presented in Fig. 11.
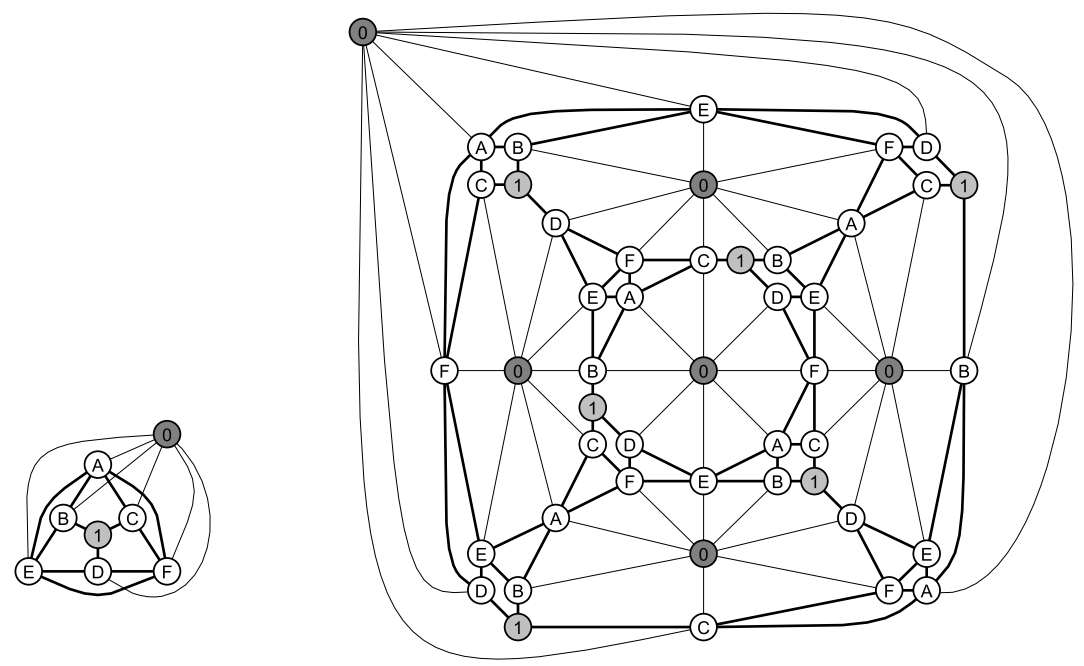

Fig. 12. Emulator for $\mathcal{B}_{7}$

Furthermore, in the same mechanical way, we can obtain planar emulators for other members of the " $K_{1,2,2,2}$-family"; namely for $\mathcal{B}_{7}, \mathcal{C}_{3}, \mathcal{D}_{2}$ in Fig. 3. Several more interesting planar emulators can be straightforwardly obtained from that of $\mathcal{E}_{2}$ by means of $Y \Delta$-transformations. See these emulators in Figures 12,13,14.

Planar emulator for $\mathcal{C}_{4}$. Consider the graph $\mathcal{C}_{4}$ drawn and labeled as in Figure 15, and observe that it is constructed of the cube graph with all nodes except for two (say 0 and 7 ) in the opposing corners of the cube adjacent to an additional vertex $x$.

Figure 16 shows the gadget we will be using: We can think of it as the trace that arises when rolling the underlying cube over its ridges. We start (north-west of the gadget) with the cube lying on the facet $\{0,1,2,3\}$, and roll it along its $\{1,3\}$ ridge, such that it lies down with the facet $\{1,5,7,3\}$. Overall, we roll the cube seven times around this axis, i.e., each possible side is downwards exactly twice; we end up at the north-east of the gadget. There, we change the roll-axis, and roll over the ridge $\{6,2\}$. Again we roll seven times and arrive at the south 

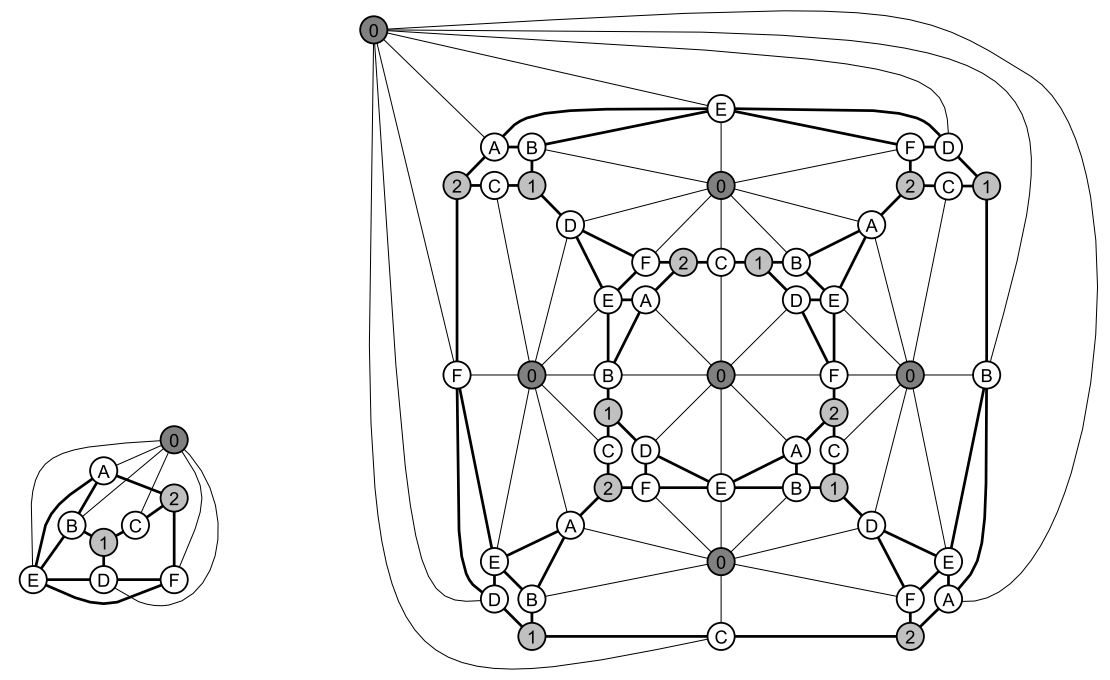

Fig. 13. Emulator for $\mathcal{C}_{3}$

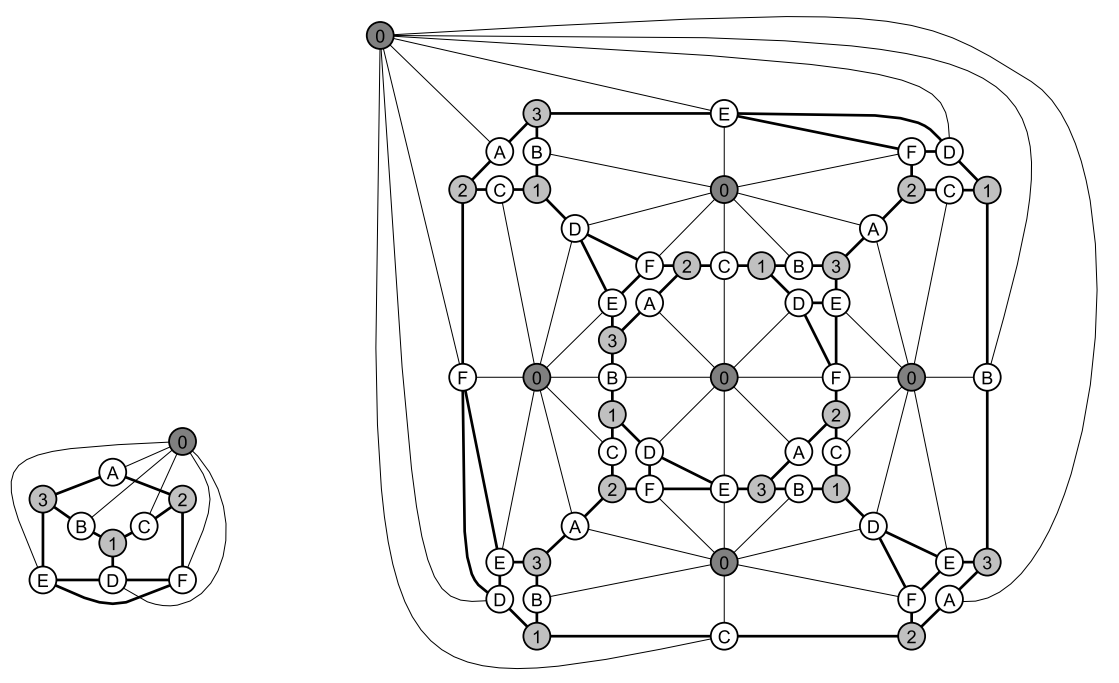

Fig. 14. Emulator for $\mathcal{D}_{2}$

of the gadget. There, we change the roll axis again, and, after seven rolls, arrive back at our start position.

The arising, triangular-shaped gadget allows an intuitive notion of outside (nodes on the outer face) and inside (all other nodes; they lie on the largest inner face). It has several important properties: 


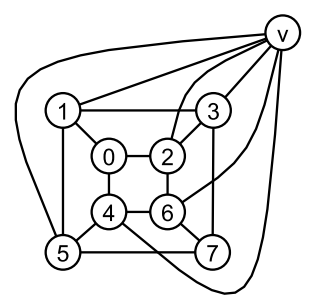

Fig. 15. The graph $\mathcal{C}_{4}$.

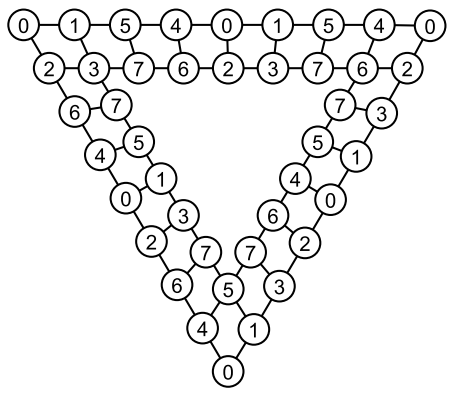

Fig. 16. Gadget used to build an emulator for $\mathcal{C}_{4}$.

- The node 0 only appears on the outside of the gadget. Each such node misses exactly one of the neighbors required for $\mathcal{C}_{4}$.

- All nodes 1-6 have degree three, and are adjacent to all the necessary neighbors (w.r.t. $\mathcal{C}_{4}$ ), except for $x$.

- The node 7 only appears on the inside of the gadget, and is adjacent to all its necessary neighbors (w.r.t. $\mathcal{C}_{4}$ ).

- Connecting all nodes on the inside (outside, respectively) of the gadget with an additional vertex $x^{\prime}$ representing $x$ suffices for $x^{\prime}$ to satisfy its emulator property for $\mathcal{C}_{4}$.

- On the outside of the gadget, the node $5(6,3)$ appears only on the north (south-west, south-east, respectively) side.

We complete the gadget by inserting a node representing $x$ into the inside of the gadget.

Now, to obtain an emulator for $\mathcal{C}_{4}$, we construct a graph embedded on a cuboctahedron (the Archimedean solid with 8 triangular and 6 square facets): clearly, we can draw its wire-frame structure planarly. Note that each of the polyhedron's ridges is neighbored by one triangular and one square facet. We label all corners of this polyhedron with 0 , and insert a (properly rotated, see below) copy of our gadget into each of the polyhedron's triangular facets. We can uniquely label the ridges of the polyhedron with 5,6 , or 3 , depending on which of these nodes appear on the gadget's side along that ridge. All nodes 0 


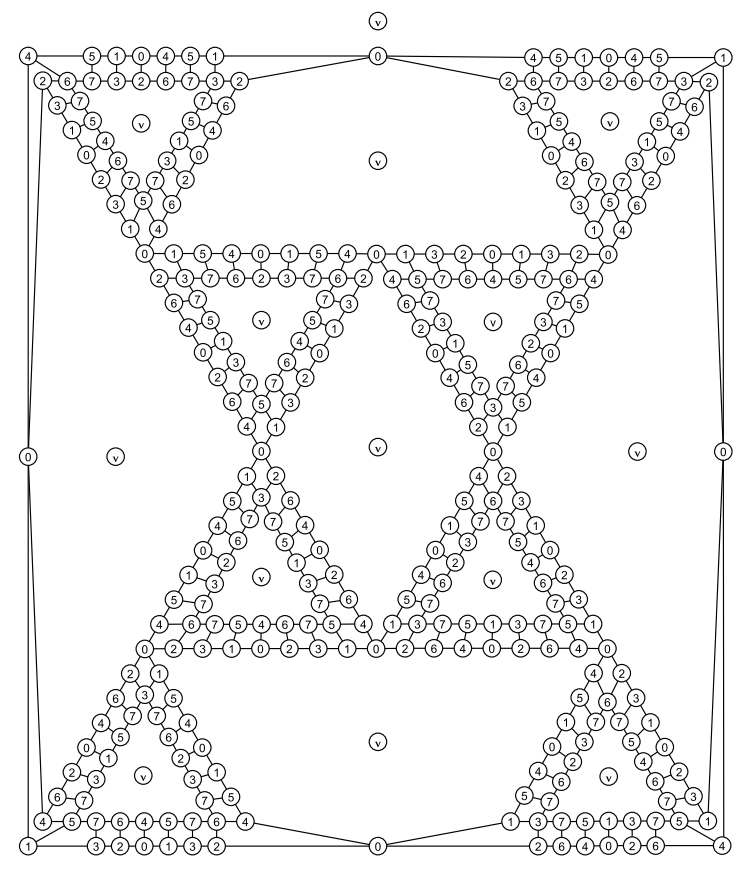

Fig. 17. The full planar emulator for $\mathcal{C}_{4}$.

have their required neighbors, and we can place a node representing $x$ into each square face and connect it with the nodes along its ridges. This establishes an emulator for $\mathcal{C}_{4}$, cf. Figure 17.

Planar emulator for $\boldsymbol{K}_{\mathbf{7}}-\boldsymbol{C}_{\mathbf{4}}$. Already the survey [10]—when commenting on the surprising Rieck-Yamashita construction - stressed the importance of deciding whether the graph $K_{7}-C_{4}$ is planar-emulable. Its importance is tied with the structural search for all potential nonprojective planar-emulable graphs; see $[11,2]$ and Section 5 for a detailed explanation. Briefly saying, $K_{7}-C_{4}$ (and its "family" of $\mathcal{D}_{3}, \mathcal{E}_{5}, \mathcal{F}_{1}$; Fig. 3) are the only projective forbidden minors which have planar emulators and are not "internally 4-connected". In fact, for several reasons we believed that $K_{7}-C_{4}$ cannot have a planar emulator, and so it came as another surprise when we have just recently discovered one.

In order to describe our planar emulator construction for $K_{7}-C_{4}$, it is useful to divide the vertex set of $K_{7}-C_{4}$ into three groups: the triple of central vertices (named 1,2,3 in Fig. 19 left) adjacent to all other vertices, and the two vertex pairs (named $A, B$ and $C, D$ ) each of which has connections only to its mate and to the central triple. This view allows us to identify a skeleton of the potential emulator as the subgraph induced on the vertices representing the 

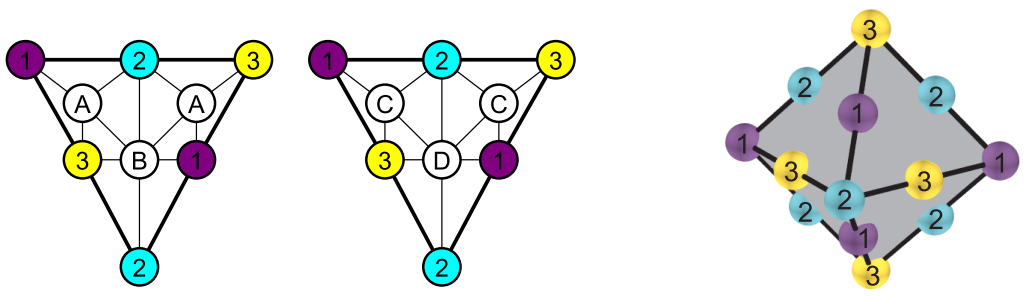

Fig. 18. Basic building blocks for our $K_{7}-C_{4}$ planar emulator: On the left, only vertex 2 misses an A-neighbor and 1,3 miss a B-neighbor. Analogically on the right. The right-most picture shows the skeleton of the emulator in a "polyhedral" manner.
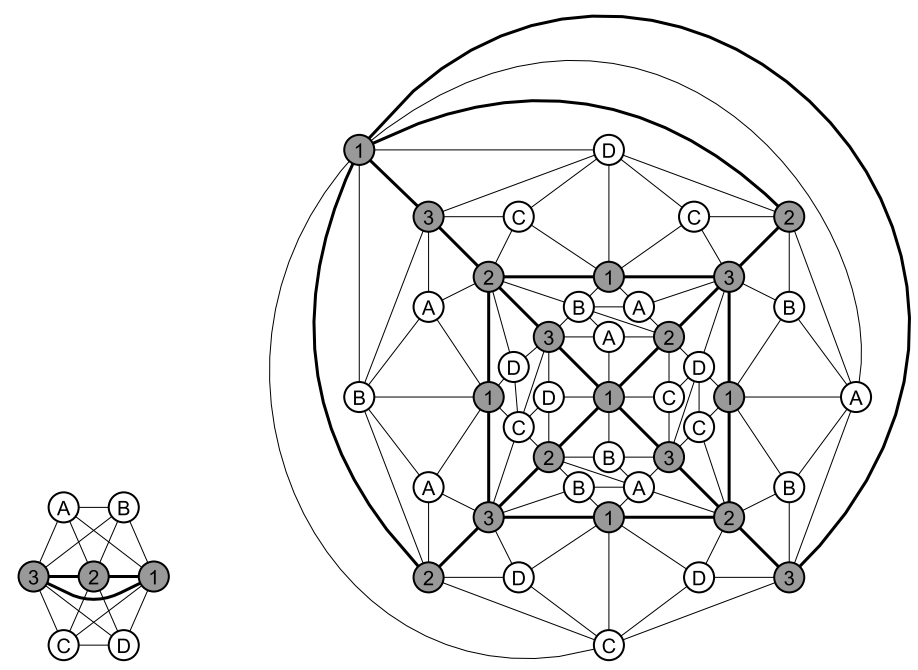

Fig. 19. A planar emulator for $K_{7}-C_{4}$, constructed from the blocks in Fig. 18. The skeleton representing the central vertices is drawn in bold.

central triple 1,2,3 and place the remaining vertices representing $A, B$ and $C, D$ into the skeleton faces, provided certain additional requirements are met.

This simple idea leads to the introduction of basic building blocks (see Figure 18), each of which "almost" emulates the subgraph induced on $1,2,3, \mathrm{~A}, \mathrm{~B}$ and $1,2,3, \mathrm{C}, \mathrm{D}$, respectively. The crucial property of the blocks is that the vertices labeled A,B or C,D have all the required neighbors in place. Finally, four copies of each of the blocks can be arranged in the shape of an octahedron such that all missing requirements in the blocks are satisfied. The resulting planar emulator is in Figure 19.

Presenting the planar emulator for $K_{7}-C_{4}$; additional notes. Emulator for $K_{7}-C_{4}$ has a similar property as the one for $\mathcal{E}_{2}$. We can embed it into a polyhedron - an octahedron in this case. We may then take 8 cells from Figure 18 
and call them $A B / C D$ cells. Three out of six outer vertices of each cell have both inner vertices as their neighbors (they are $A B$ or $C D$-satisfied) and the remaining three have one (they are $A B$ or $C D$-half-satisfied). We take four cells of each kind and join the outer vertices such that every $A B / C D$ cell will represent one facet of an octahedron and no two cells of the same kind will be adjacent. Notice that the central vertices in the middle of each ridge of the octahedron are incident to two facets and central vertices on the corners of the octahedron are incident to four facets. We now rotate the $A B / C D$ cells such that vertices on corners are twice $A B$-half-satisfied and twice $C D$-half-satisfied, every time by a different vertex, and vertices on the ridges are $A B$-satisfied by one of two incident facets and $C D$-satisfied by the other. Such a construction is an emulator for $K_{7}-C_{4}$ and can surely be drawn planarly (see Figure 19).

Planar emulator for $\mathcal{D}_{3}$. Once we can emulate $K_{7}-C_{4}$, the natural question to ask is if this construction can be extended to $\mathcal{D}_{3}$, a graph which is created by applying a single $\Delta Y$ transformation on $K_{7}-C_{4}$ (replacing one triangular face of $K_{7}-C_{4}$ by a vertex of degree 3 ) - see Figure 20. Clearly, the same construction does not work, because of a special property of vertex 1 , which will be discussed later. Again, we call again three vertices labeled 1,2,3 the central vertices and two other components $A B C$ and $D E$. We will consider an extra edge between vertices 1 and 3 , because it does not influence the property of having an emulator in this case (see section 5).

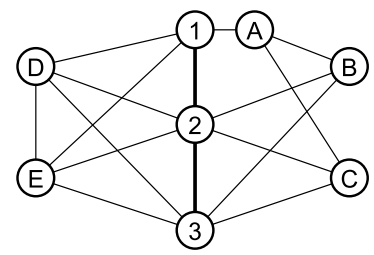

Fig. 20. $\mathcal{D}_{3}$

While building $K_{7}-C_{4}$ we used two triangular gadgets $A B$ cell and $C D$ cell. If we upgrade one of them in order to get a cell suitable for the $A B C$ component of $\mathcal{D}_{3}$ (a cell with central vertices as outer vertices, satisfying all the inner and the maximum of outer vertices) and try to establish the emulator in the exactly same way as for $K_{7}-C_{4}$ (using an octahedron), we arrive at a single, but fatal obstruction - the vertex 1 cannot be half-satisfied by the $A B C$ cell, simply because it only has one neighbor among $\mathrm{A}, \mathrm{B}$ and $\mathrm{C}$. Therefore no vertex 1 on the corner of an octahedron can meet its requirements. Nevertheless, we can take a $K_{7}-C_{4}$ emulator as a core and "fix" the properties of such vertices.

Let's have two building blocks as in the $K_{7}-C_{4}$ case and define two other supporting cells, $A B C$-small-cell and DE-small-cell (see Figure 21). These will 


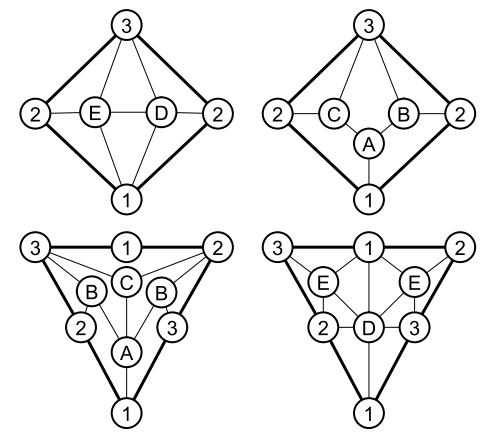

Fig. 21. Building blocks for $\mathcal{D}_{3}$ emulator.

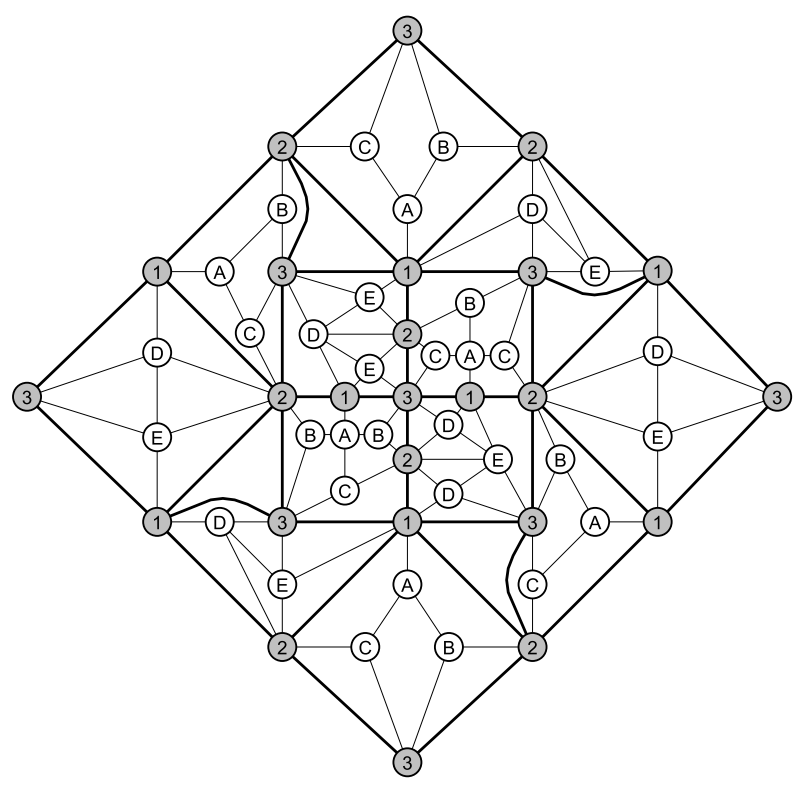

Fig. 22. The construction built with one half of the emulator for $K_{7}-C_{4}$ and 8 small cells for the outer vertices to have the maximal number of different neighbors.
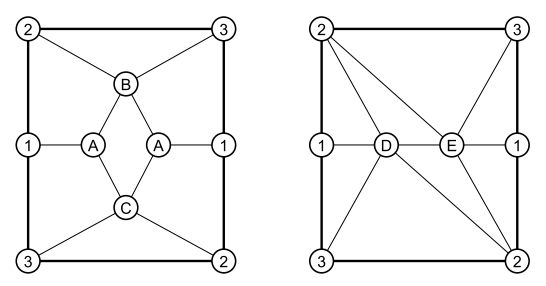

Fig. 23. The hexagonal cell for connecting two identical components from Figure 22 into an $\mathcal{D}_{3}$ emulator. 


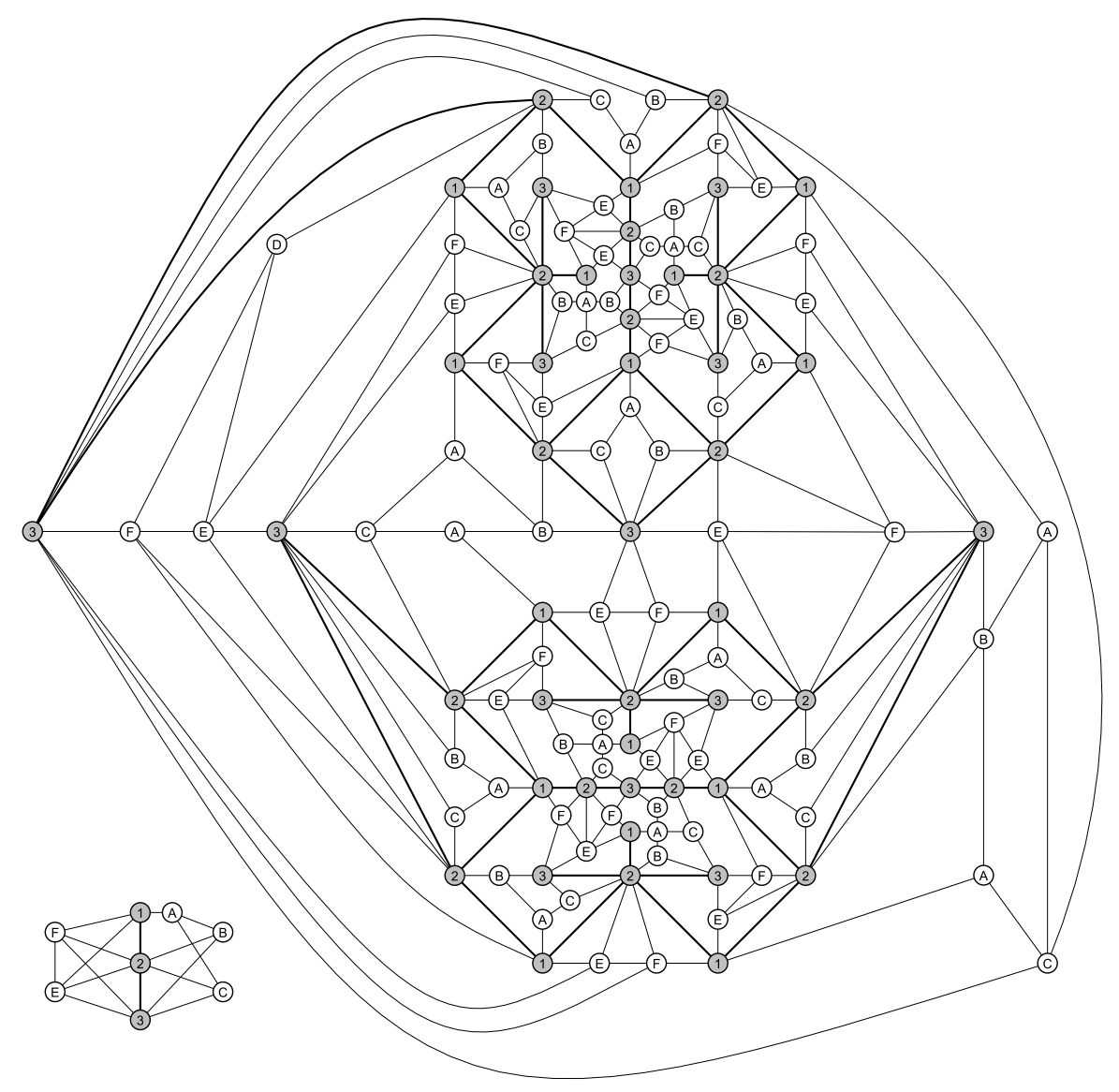

Fig. 24. The finite planar emulator for $\mathcal{D}_{3}$.

help us overcome the above mentioned drawback. We take one half of the emulator for $K_{7}-C_{4}$ and upgrade the two $A B$ cells to $A B C$ cells. Now we surround the graph with four $A B C$-small-cells and four $D E$-small-cells, such that the central vertices have all desired neighbors among $\{1,2,3\}$ and the outer vertices of the new expanded graph have better properties concerning the number and kind of neighbors (see Figure 22). We can observe that all vertices labeled with 3 are $A B C$-satisfied or DE-satisfied, but do not have one of the central vertices as a neighbor, vertices labeled with 1 are $A B C$-satisfied and DE-half-satisfied or $D E$-satisfied and vertices with label 2 are DE-satisfied and $A B C$-half-satisfied or $A B C$-satisfied. Additionally, if there is an edge between marginal vertex 1 and 2 , then they miss some neighbors from the component of the same kind. This fact enables us to copy the whole graph in Figure 22 and join it in a smart way with the original graph to obtain an emulator. If we identify pairs of vertices with label 3 from those two copies (such that vertices that have only vertices 1 as a 


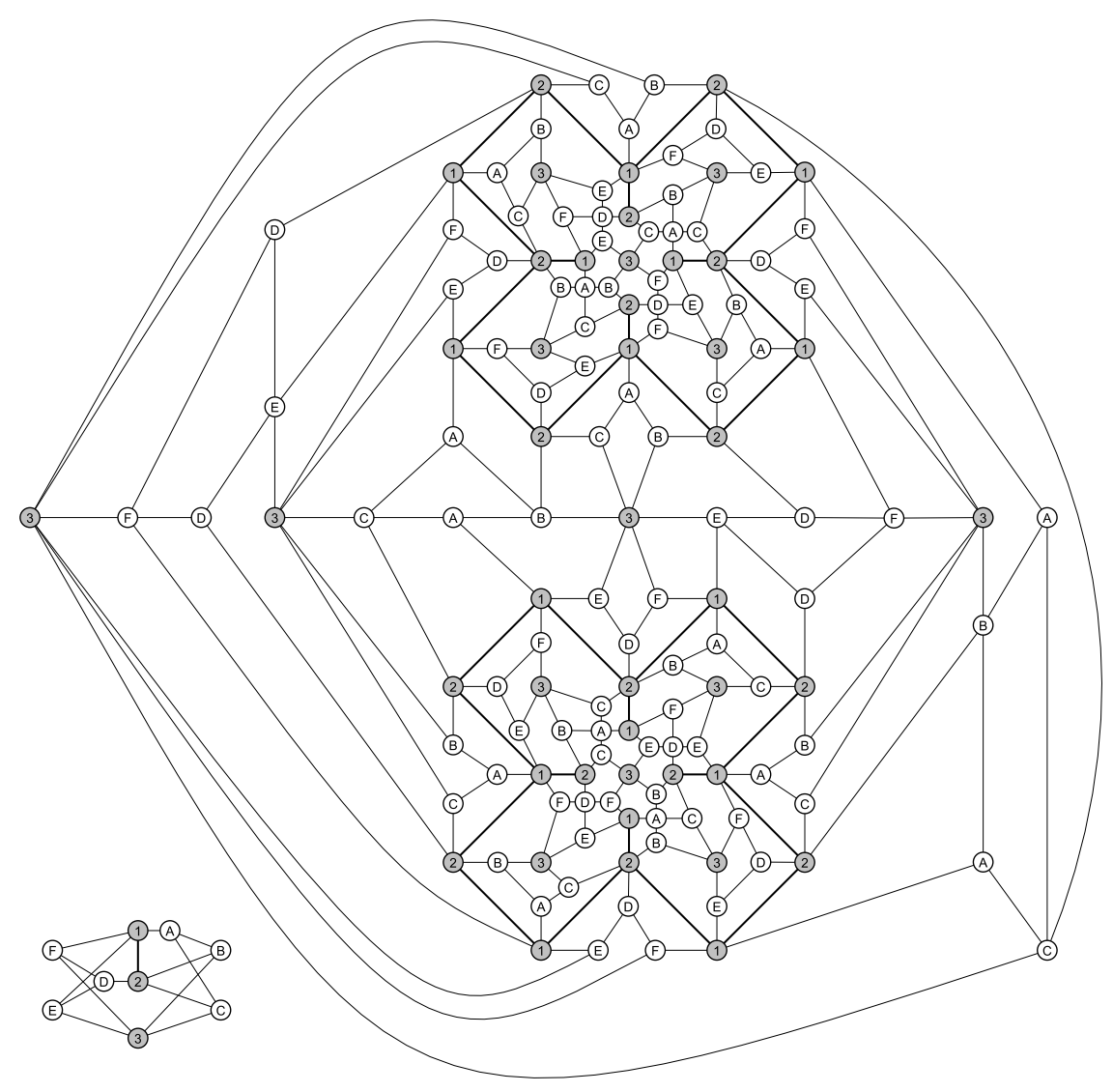

Fig. 25. The finite planar emulator for $\mathcal{F}_{1}$

neighbor will be identified with the vertex which have the opposite problem), we get 4 empty hexagons, whose borders are made of two vertices with label 3 , which are completely satisfied, two vertices with label 1, which are $A B C$-satisfied and DE-half-satisfied and two vertices with label 2, which are $A B C$-satisfied (it holds for two of those hexagons, for the other two the $A B C$ and DE-satisfactory properties are switched). Thus these hexagons can be easily filled with the simple pattern for the border vertices to meet the required conditions (see Figure 23).

The final emulator for $\mathcal{D}_{3}$ is presented in Figure 24 and the above described approach is clearly visible (two identical components derived from an emulator for $K_{7}-C_{4}$ connected together).

Planar emulator for $\mathcal{F}_{1}$. The construction of an emulator for $\mathcal{F}_{1}$ follows the same pattern as building an emulator for $\mathcal{D}_{3}$. In fact, the emulator for $\mathcal{F}_{1}$ was found first by the above mentioned construction and the emulator for $\mathcal{D}_{3}$ resulted 

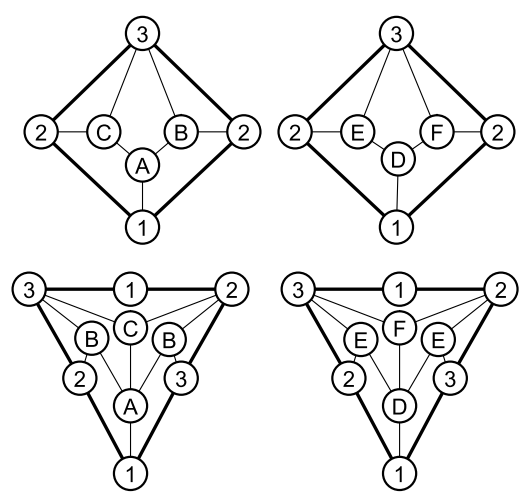

Fig. 26. Building cells for $\mathcal{E}_{5}$ emulator

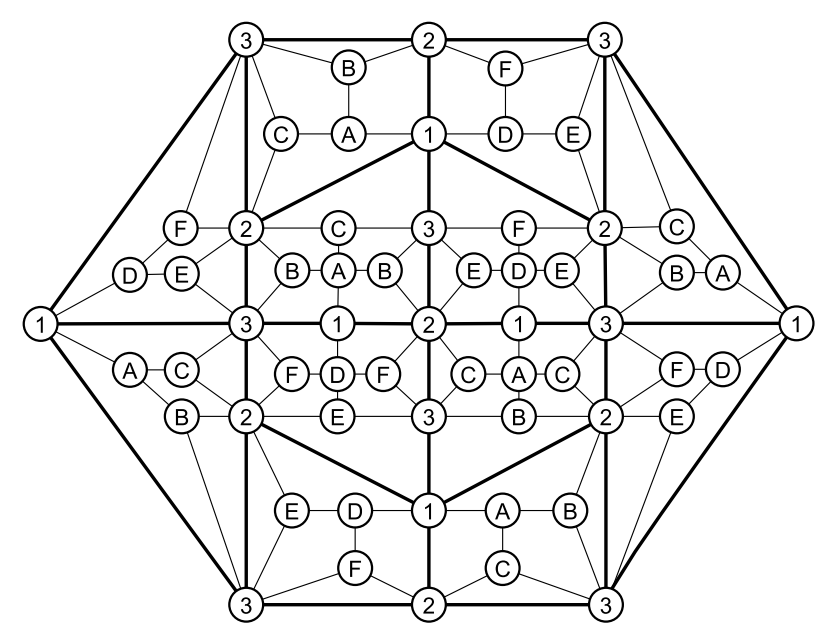

Fig. 27. The construction for $\mathcal{E}_{5}$ built upon a "half" of a $K_{7}-C_{4}$ emulator and 8 small cells for the outer vertices to have the best possible properties

from a simplification of an emulator for $\mathcal{F}_{1},\left(\mathcal{D}_{3}\right.$ results from $\mathcal{F}_{1}$ by taking a $Y \Delta$ transformation, which is trivial to perform in the emulator). Therefore we present only the final emulator picture (see Figure 25).

Planar emulator for $\mathcal{E}_{5}$. In order to obtain an emulator for $\mathcal{E}_{5}$, we again take one half of the emulator for $K_{7}-C_{4}$ (as in the $\mathcal{D}_{3}$ case) and replace $A B / C D$ cells by $A B C / D E F$ cells. Let's call this construction a core. As in $\mathcal{D}_{3}$ case, we consider an additional edge 13, which is not present in $\mathcal{E}_{5}$ but makes the pictures easier to understand. Similarly, we use some smaller additional cells to improve the properties of the outer vertices of the core (see Figure 26). Since $\mathcal{E}_{5}$ is slightly 


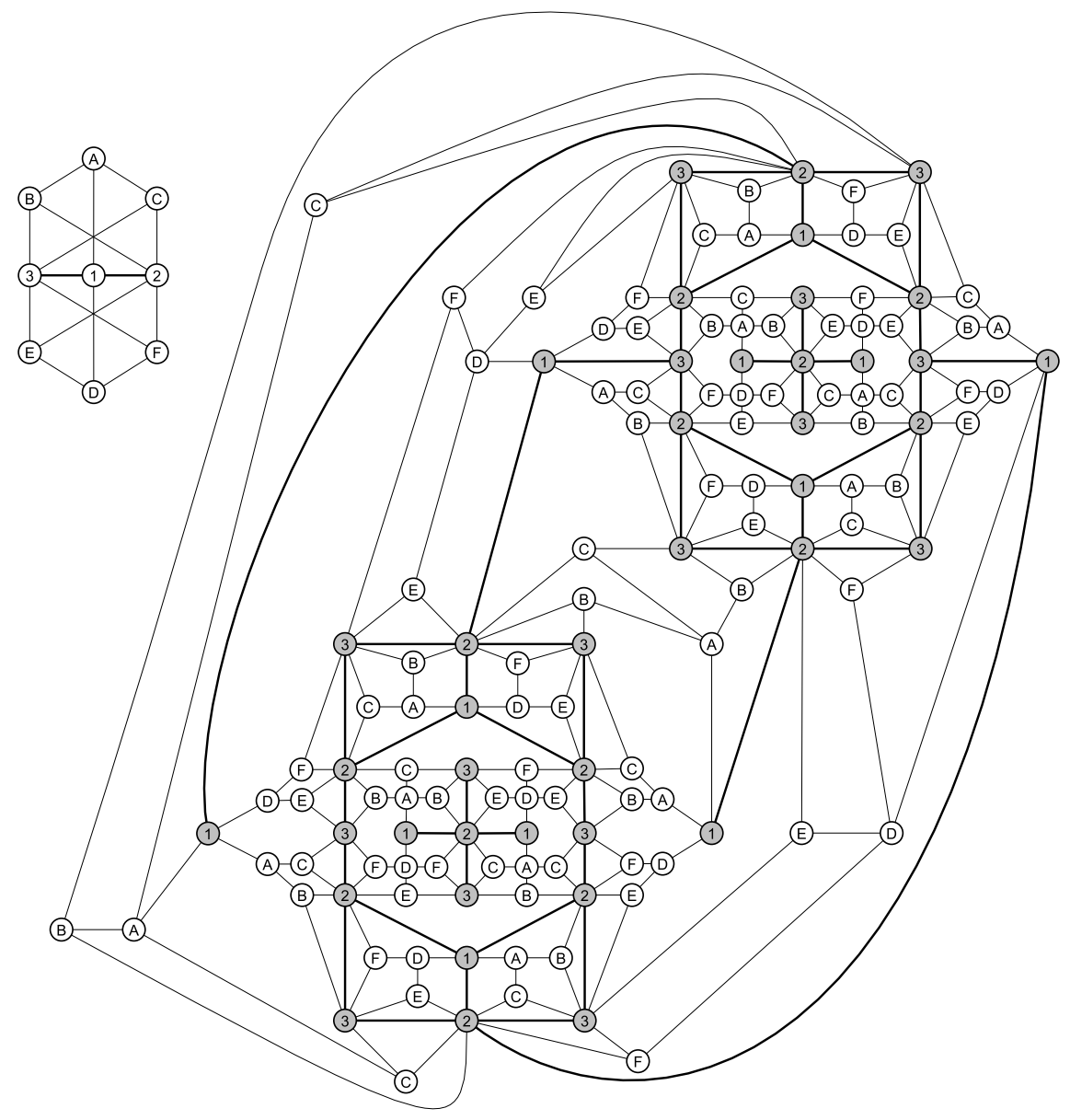

Fig. 28. The finite planar emulator for $\mathcal{E}_{5}$

different from $\mathcal{F}_{1}$ (both come from $K_{7}-C_{4}$, but two $\Delta Y$ transformations took place in different triangle faces, so there exists a vertex (labeled 2) in $\mathcal{E}_{5}$ which is adjacent to two new vertices of degree 3 , but is not present in $\mathcal{F}_{1}$ ), the use of the supporting small cells is quite different as well. We surround the core as showed in Figure 27. In this way we arrive to better properties of the outer vertices of the new graph. Outer vertices labeled 1 are completely satisfied, vertices 2 are half-ABC-satisfied and half-DEF-satisfied and vertices 3 are $A B C$-satisfied and $D E F$-half-satisfied or vice versa.

Now we use a similar tool as in the previous cases of $\mathcal{D}_{3}$ and $\mathcal{F}_{1}$ - we duplicate the graph and connect the two copies in a clever way so that vertices 1 get the desired neighbor 2 as well and four new hexagons are created. The vertices surrounding each hexagonal face are missing some neighbors of the same com- 
ponent. We fill each face with a $A B C$ or $D E F$ cell to satisfy all the remaining vertices. Now we have a complete emulator for $\mathcal{E}_{5}$ (see Figure 28).

\section{Structural Search: How far can we go?}

Until now, we have presented several newly discovered planar emulators of nonprojective graphs. Unfortunately, despite the systematic construction methods introduced in Section 4, we have got nowhere closer to a real understanding of the class of planar-emulable graphs. It is almost the other way round - the new planar emulators evince more and more clearly how complicated the problem is. Hence, we also need to consider a different approach.

The structural search method, on which we briefly report in this section, is directly inspired by previous [11]; we refer to $[2,3]$ for closer details which cannot fit into this paper.

The general idea can be outlined as follows: If $H$ is a mysterious nonprojective planar-emulable graph, then $H$ must contain one of the projective forbidden minors, say $F$, while $F$ cannot be among those forbidden minors not having planar emulators (Theorems 3.4, 3.6). Now there are basically three mutually exclusive possibilities:

i. $H$ is a planar expansion of a smaller graph. A graph $H$ is a planar expansion of $G$ if it can be obtained by repeatedly substituting a vertex of degree $\leq 3$ in $G$ by a planar subgraph with the attachement vertices on the outer face.

ii. $H$ contains a nonflat 3-separation. A separation in a graph is called flat if one of the sides has a plane drawing with all the boundary vertices on the outer face.

iii. $H$ is internally 4-connected, i.e., it is 3-connected and each 3-separation in $H$ has one side inducing the subgraph $K_{1,3}$ (informally, $H$ is 4-connected up to possible degree-3 vertices with stable neighborhood).

We denote by $\left\langle K_{7}-C_{4}\right\rangle=\left\{K_{7}-C_{4}, \mathcal{D}_{3}, \mathcal{E}_{5}, \mathcal{F}_{1}\right\}$ the family of $K_{7}-C_{4}$. The underlying idea is that all the graphs in a family are $Y \Delta$-transformable to the family's base graph. Particulary the family of $K_{7}-C_{4}$ comprises all the projective forbidden minors in question which are not internally 4-connected. See in Fig. 3.

In the case (i.) above, we simply pay attention to the smaller graph $G$. In the case (ii.), one can argue that either the projective forbidden minor $F$ (in $H$ ) itself contains a nonflat 3-separation (so $F \in\left\langle K_{7}-C_{4}\right\rangle$ ), or $F$ is internally 4-connected and $H$ then is not planar-emulable (a contradiction). The former is left for further investigation. Finally, in the case (iii.) we may apply a socalled splitter theorem for internally 4-connected graphs [13], provided that $F$ is also internally 4-connected. This leads to a straightforward computerized search which has a high chance to finish in finitely many steps, producing all such desired internally 4-connected graphs $H$.

Actually, when the aforementioned procedure was applied to the planar cover case in [11], the search was so efficient that the outcome could have been de- 
scribed by hand; giving all 16 specific graphs that potentially might be counterexamples to Conjectures 1.1. In our emulator case, we get the following:

Theorem 5.1 ([3]). Let $H$ be a nonprojective planar-emulable graph. Then, $H$ is a planar expansion of one of specific 175 internally 4-connected graphs, or $H$ contains a minor isomorphic to a member of $\left\{\mathcal{E}_{2}, K_{4,5}-4 K_{2}\right\} \cup\left\langle K_{7}-C_{4}\right\rangle$.

Up to this point, we have not been successful in finishing the computations for the graphs $F=K_{4,5}-4 K_{2}$ and $\mathcal{E}_{2}$, due to the high complexity of the generated extensions. Yet, we strongly believe that it is possible to obtain finite results also for those cases, perhaps with the help of an improved generating procedure. On the other hand, the cases starting with $F \in\left\langle K_{7}-C_{4}\right\rangle$ will need an alternative procedure, e.g., using so-called "separation bridging". This is subject to future investigations.

\section{Conclusion and Further Questions}

While our paper presents new and surprising findings about planar-emulable graphs, the truth is that these findings are often negative in the sense that they bring more intriguing questions than answers. Of course, the fundamental open question in the area is to find a characterization of the class of planaremulable graphs in terms of some other natural (and preferably topological) graph property. Even coming up with a plausible conjecture (cf. Conjecture 1.1) would be of high interest, but, with our current knowledge, already this seems to be out of reach yet.

Instead, we suggest to consider the following specific (sub)problems:

- Is there a planar emulator of the graph $K_{4,4}-e$ ? We think the answer is no, but are currently unable to find a proof, e.g. extending the arguments of [7].

- The emulators shown in Section 4 suggest that we can, in some mysterious way, reflect $\Delta Y$-transformations in emulator constructions (i.e., the converse direction of Proposition 3.2). Such a claim cannot be true in general since, e.g., a $Y \Delta$-transformation of the graph $\mathcal{D}_{4}$ (Fig. 3) leads to a strict subgraph of $\mathcal{B}_{3}$, which therefore has a two-fold planar cover while $\mathcal{D}_{4}$ is not planaremulable by Theorem 3.4. But where is the precise breaking point?

- The two smallest projective forbidden minors are on 7 vertices, $K_{7}-C_{4}$ (missing four edges of a cycle) and $K_{1,2,2,2}$ (missing three edges of a matching). Both of them, however, have planar emulators while their common supergraph $K_{7}$ does not. What is a minimal subgraph of $K_{7}$ not having a planar emulator? Can we, at least, find a short argument that the graph $K_{7}-e$ has no planar emulator?

- Finally, Conjecture 1.1 can be reformulated in a way that a graph has a planar cover iff it has a two-fold planar cover. The results of [11] moreover imply that the minimal required fold number for planar-covers is bounded by a constant. Although, in the emulator case, the numbers of representatives for each vertex of the emulated graph differ, there is still a possibility 
of a fixed upper bound on them: Is there a constant $K$ such that every planar-emulable graph $H$ has a planar emulator with projection $\psi$ such that $\left|\psi^{-1}(v)\right| \leq K$ for all $v \in V(H)$ ? A computerized search as in Section 5 would be of great help in this task.

\section{References}

1. D. Archdeacon, A Kuratowski Theorem for the Projective Plane, J. Graph Theory 5 (1981), 243-246.

2. M. Derka, Planar Graph Emulators: Fellows' Conjecture, Bc. Thesis, Masaryk University, Brno, 2010. http://is.muni.cz/th/255724/fi_b/thesis.pdf

3. M. Derka, Towards Finite Characterization of Planar-emulable Non-projective Graphs, Congressus Numerantium 207-211 (2011), submitted.

4. M. Fellows, Encoding Graphs in Graphs, Ph.D. Dissertation, Univ. of California, San Diego, 1985.

5. M. Fellows, Planar Emulators and Planar Covers, Unpublished manuscript, 1988.

6. H. Glover, J.P. Huneke, C.S. Wang, 103 Graphs That Are Irreducible for the Projective Plane, J. of Comb. Theory Ser. B 27 (1979), 332-370.

7. P. Hliněný, $K_{4,4}-$ e Has No Finite Planar Cover, J. Graph Theory 27 (1998), 51-60.

8. P. Hliněný, Planar Covers of Graphs: Negami's Conjecture, Ph.D. Dissertation, Georgia Institute of Technology, Atlanta, 1999.

9. P. Hliněný, Another Two Graphs Having no Planar Covers, J. Graph Theory 37 (2001), 227-242.

10. P. Hliněný, 20 Years of Negami's Planar Cover Conjecture, Graphs and Combinatorics 26 (2010), 525-536.

11. P. Hliněný, R. Thomas, On possible counterexamples to Negami's planar cover conjecture, J. of Graph Theory 46 (2004), 183-206.

12. J.P. Huneke, A Conjecture in Topological Graph Theory, In: Graph Structure Theory (Seattle, WA, 1991), N. Robertson and P.D. Seymour editors, Contemporary Mathematics 147 (1993), 387-389.

13. T. Johnson, R. Thomas, Generating Internally Four-Connected Graphs, J. Combin. Theory Ser. B 85 (2002), 21-58.

14. M. Klusáček, Construction of planar emulators of graphs, Bc. Thesis, Masaryk University, Brno, 2011. http://is.muni.cz/th/324101/fi_b/bc_thesis.pdf

15. S. Negami, Enumeration of Projective-planar Embeddings of Graphs, Discrete Math. 62 (1986), 299-306.

16. S. Negami, The Spherical Genus and Virtually Planar Graphs, Discrete Math. 70 (1988), 159-168.

17. S. Negami, Graphs Which Have No Finite Planar Covering, Bull. of the Inst. of Math. Academia Sinica 16 (1988), 378-384.

18. Y. Rieck, Y. Yamashita, Finite planar emulators for $K_{4,5}-4 K_{2}$ and $K_{1,2,2,2}$ and Fellows' Conjecture, European Journal of Combinatorics 31 (2010), 903-907. 